\title{
The Effect of Entity Generalization on Software Functional Sizing: A Case Study
}

\author{
Oktay Turetken ${ }^{1, *}$, Onur Demirors ${ }^{1}$, Cigdem Gencel ${ }^{2}$, Ozden Ozcan Top ${ }^{1}$, \\ and Baris Ozkan ${ }^{1}$ \\ ${ }^{1}$ Informatics Institute, Middle East Technical University, 06531, Ankara, Turkey \\ \{oktay, demirors, ozden, bozkan\}@ii.metu.edu.tr \\ ${ }^{2}$ Blekinge Institute of Technology, Department of Systems and Software Engineering \\ cigdem.gencel@bth.se
}

\begin{abstract}
In this paper we discuss a specific result derived from a multiple case study. The case study involved implementation of IFPUG Function Point Analysis and COSMIC Functional Size Measurement methods in an innovative military software development project by different groups of experts. Application of these methods in a case that provides a number of size measurement challenges enabled us to observe significant improvement opportunities for the methodologies. In this paper, we depict the utilization of the entity generalization concept in two FSM methods and based on our observations we discuss the effects of different interpretations of the concept for measuring the software functional size.
\end{abstract}

Keywords: Functional size measurement, COSMIC FSM, IFPUG FPA, entity generalization.

\section{Introduction}

Poor estimation remains to be one of the main reasons for software project failures. Functional Size Measurement (FSM) methods are advocate for providing necessary input for estimation models. FSM methods are intended to measure the size of software by quantifying the functionality delivered to the user. Since the introduction of the concept [2], a variety of FSM methods have been formulated and many improvements have been made on these methods [11].

FSM methods have their own definition of functionality, utilize different counting rules for the different functional components of functional user requirements and have their own units and scales for their measures. In spite of these differences, they are expected to produce similar results as they are based on a set of shared principles. A number of research studies have been performed in order to clarify their conceptual basis and establish the common principles of FSM methods [8], [10], [13].

This study is supported by The Scientific and Technological Research Council of Turkey (TUBITAK), Project 107E010. 
The objectives of this paper are to discuss how the concept of entity generalization is considered in commonly used FSM methods; the International Function Point Users Group Function Point Analysis (IFPUG FPA) [17] and the Common Software Measurement International Consortium FSM (COSMIC FSM) [18] and to investigate how different interpretations of this concept affect the functional size of the software measured by these methods. Findings are based on the case study we conducted on an innovative military software development project. Specifically, we observe how entity abstractions - in the form of inheritance or generalization/specialization between entities or classes - may lead to different assumptions when identifying elementary components for the measurement and the effects of these different assumptions on the functional size. We evaluate the methods based on our findings and discuss the difficulties we faced during the implementation of the methods.

The paper is organized as follows: Section 2 briefly summarizes the FSM methods and related research. In section 3, we describe the case study. Section 4 presents our findings and conclusions.

\section{Related Work}

Measuring the software size with the 'functionality' attribute was first introduced by Albrecht [2] in his Function Point Analysis (FPA) method. With the refinements of the technique, FPA has evolved into the IFPUG FPA [12] method. During the following years, several new measurement methods ([1], [5], [22], [29]) or extending the applicability of FSM methods to different functional domains in addition to business application software ([21], [28], [30]) have been developed. Studies by Symons' [26] and Gencel et al. [11] provide detailed discussions on FSM methods.

The publication of the first ISO/EIC's 14143-1 standard [14] in 1998 aimed at clarifying the fundamental concepts of FSM. It defined concepts such as 'Functional User Requirements (FUR) ${ }^{1}$, ' 'Functional Size ${ }^{2}$, , Base Functional Component (BFC) ${ }^{3}$, 'BFC Type' and the FSM requirements that should be met by a candidate method. Currently, Mark II FPA (MkII FPA) [16], IFPUG FPA [17], COSMIC FSM [18] and Netherlands Software Metrics Association FSM (NESMA FSM) [19] are certified by ISO as being international standards.

Earlier FSM methods have been criticized of lacking support for concepts such as inheritance and aggregation [1], [7], [24], which are generally associated with objectoriented methodologies. This creates ambiguities and difficulties in determining the functional components and measuring the functional size.

In order to better reflect the needs of object-oriented (OO) software development methodologies, several approaches have been proposed. Some of these works that

${ }^{1}$ FURs: A sub-set of the user requirements. The FURs represent the user practices and procedures that the software must perform to fulfill the users' needs.

2 Functional Size: A size of the software derived by quantifying the FUR.

${ }^{3}$ BFC: An elementary unit of FUR defined by and used by an FSM Method for measurement purposes.

${ }^{4}$ BFC Type: A defined category of BFCs. A BFC is classified as one and only one BFC Type. 
adapt FPA method to OO concepts yield results that are similar to what would have been obtained by directly applying IFPUG FPA. Whitmire [31] considered each general class as a logical file and methods sent across the application boundary as transactional functions. For classes that are part of an inheritance hierarchy, "if the generalization is truly part of the application domain, it is counted as a separate logical file". If the generalization was build for the ease of modeling, general class is counted with each specialized class. In IFPUG FPA, a logical file is a user identifiable group of logically related data or control information. Internal Logical Files (ILFs) are maintained within the boundary of the application, whereas External Logical Files (EIFs) are maintained within the boundary of another application.

Fetcke et al. [7] defined rules for mapping OO-Jacobson method to concepts from IFPUG FPA and verified the rules by applying them in three case studies. For inheritance relationships, they defined two rules. First; an abstract class is not visible to the user and does not relate logical files itself. It is rather a candidate for a record element type (RET) for each class that inherits its properties. RETs are optional or mandatory subgroup of data elements within an ILF or EIF. They influence the degree of functional complexity (low, average, high) of logical files. Second; specialized classes of a concrete general class are candidates for a logical file or a RET of that class. With these presumptions, however, the work does not elucidate whether specialized classes are logical files of their own or RETs for the general class.

To overcome these difficulties Abrahao et al. [1] proposed OO-Method Function Points (OOmFP) for measuring the functional size of $\mathrm{OO}$ systems which is compliant with the IFPUG FPA rules.

Similarly, Caldiera et al. [3] adapted IFPUG FPA rules for measuring object oriented analysis and design specifications. They proposed alternative ways for identifying logical files and handling entity abstractions, but did not propose clear rules or recommendations of when and under what conditions each can be applied. In an inheritance hierarchy, a logical file may comprise all the entities in the hierarchy or every entity can be mapped to a logical file.

Mapping of object oriented modeling concepts onto the measurement constructs has also been studied for COSMIC FSM. Jenner [20] proposed a mapping for the concepts used in UML diagrams onto the abstract COSMIC FSM model. It is argued that UML sequence diagrams have a more appropriate level of granularity to measure functional size. Diab et al. [6] proposed a set of formal rules for applying COSMIC FSM to object-oriented specifications. The work proposes a formalization of the COSMIC FSM measure for the real-time object oriented modeling language.

\section{The Case Study}

We conducted a multiple-case study in order to evaluate and explore the similarities and differences between FSM methods. By implementing IFPUG FPA [12] and COSMIC FSM [28] methods, different measurers measured the functional size of a case project. 
In the scope of this paper, we deal only with the differences among methods in handling the entity generalization and how these affect the measurement results between the functional size figures obtained by different measurers. Therefore, although the description of the whole case study is presented; in this paper, the results which are related to these specific questions on entity generalization are discussed. It should be noted that our aim here is not to find out which of the methods is better, but to shed light into the improvement opportunities of each of these methods.

\subsection{FSM Methods Utilized}

In general, FSM methods first requires the functional user requirements (FUR) to be decomposed into 'Transactions', which involve inputting, outputting and processing of items or groups of data, triggered by events outside the software [15]. From transactions, BFCs are identified and then each of these is categorized to BFC Types and the attributes relevant for obtaining the base counts are identified. The next step is the actual measurement where the functional size of each BFC is measured by applying a measurement function to the BFC Types and the related attributes. The overall functional size of the software system is computed by summing up the results.

In IFPUG FPA, the BFCs are classified as the Transactional Function (TF) Types and Data Function (DF) Types. DF may be an Internal Logical File (ILF) or an External Interface File (EIF), whereas a TF can be of the type; External Input (EI), External Output (EO), or External Inquiry (EQ). These components are weighted according to their complexity and their weights are summed. The functional complexity of each logical file is based on the number of record element types (RETs) (subgroup within a logical file) and the number of data element types (DETs) within a logical file. A DET is a unique user recognizable, non-repeated field which is equivalent to the 'entity attribute'. The functional complexity of a logical file can be low, average or high, each corresponding to an IFPUG function point value.

In COSMIC FSM each FUR is decomposed into 'Functional Processes' (FP) and each of these FPs is assumed to comprise a set of sub-processes, called Data Movement Types. Data movement types are the BFCs of this method. A data movement moves one or more data attribute belonging to a single 'data group', where each included data attribute describes a complementary aspect of the same 'object of interest'. An object of interest is any 'thing' or a conceptual object that is identified from the point of view of the Functional User Requirements. It is equivalent to 'entity-type' in entity relationship (ER) analysis or 'class' in UML [23]. There are four kinds of Data Movement Types: Entry (E), Exit (X), Read (R), and Write (W). Each of these is defined as a BFC Type [18]. The value in COSMIC FP is the total number of data movements performed in the software system.

A detailed discussion on the differences and similarities between these two methods can be found in [10].

\subsection{Description of the Case Project and the Software Application}

The case project involved the development of a conceptual modeling tool (KAMA) that provides a common notation and a method for the conceptual model developers 
in different modeling and simulation development projects particularly in the military domain. The project was started in June 2005 and completed in July 2007.

The total number of project staff worked consisted of 21 people; 1 project manager, 1 assistant project manager, 2 steering committee members, 1 project coordinator, 8 researchers, 1 software development team leader, 1 quality assurance team leader, 4 software engineers (1 part-time), 1 part-time test engineer and 2 quality engineers ( 1 part-time). The efforts utilized for the project totaled up to 1,832 persondays. Table 1 gives the details of the efforts utilized for the tool development part of the project.

Table 1. The development effort for the case project

\begin{tabular}{lc}
\hline $\begin{array}{l}\text { Software Development } \\
\text { Life Cycle Phase }\end{array}$ & $\begin{array}{l}\text { Effort } \\
\text { (person-days) }\end{array}$ \\
\hline Development Processes & 1,287 \\
Software Requirements Analysis & 227 \\
Software Design & 185 \\
Software Coding \& Unit Testing & 670 \\
Testing & 205 \\
Management & 135 \\
Supporting Processes & 410 \\
Total & $\mathbf{1 , 8 3 2}$ \\
\hline
\end{tabular}

The types of software tools and programming languages used in the development phases were as follows: Rational Software Architect as the software analysis and design tool, Requisite Pro as the requirements management tool, and $\mathrm{C \#}$ as the programming language. Unified Modeling Language (UML) [23] was used for representing analysis and design. Related IEEE standards were utilized for the project work products, which were kept under configuration control by the Subversion tool.

With respect to CHAR Method defined in [15], the functional domain of the KAMA is determined as 'Information System'.

KAMA is a graphical modeling tool that supports a specific notation based on UML. It supports the development of conceptual models with a set of diagrams, model elements and their relationships. Each diagram simply consists of a set of model elements and the relationships between them. The type of model elements in a diagram and the type of the relationships that can exist between them is determined by the diagram type. The notation comprises 8 diagram types, 10 model element types and 15 relationship types. The diagram entity has a common set of attributes maintained for all types. For the model elements, on the other hand, together with the common attributes that are maintained by all, there exist attributes specific to types. Similar situation also holds for relationship types. Fig. 1 depicts the model element, relationship and diagram entities and partial data model for the entity abstractions with an extended entity-relationship (EER) model.

The characteristics of the data entities to be maintained by the tool make it a good candidate for generalization/specialization practices to be applied for model element, diagram and relationship entities. 
a)

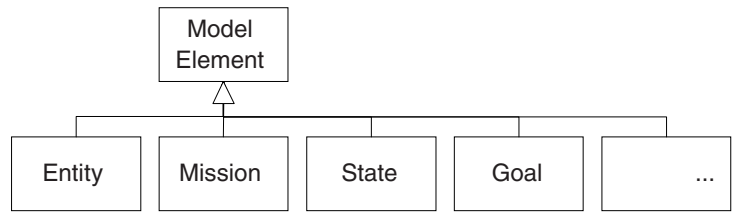

Relationship

b)

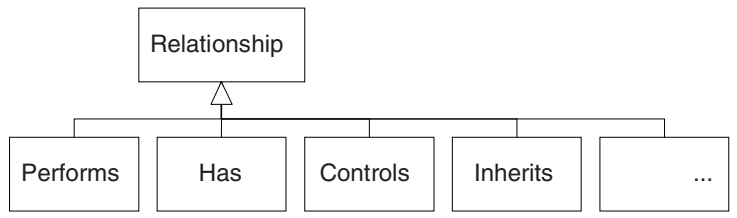

c)

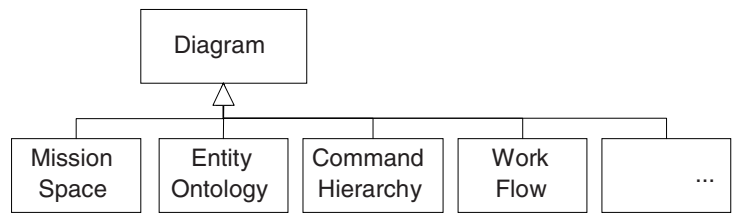

Fig. 1. Entity generalizations for diagrams, model elements and relationships

\subsection{Case Study Conduct}

The functional size measurement of the KAMA was performed by IFPUG FPA and COSMIC FSM based on the software requirements specification (SRS) document. Together with the requirements statements, the document included UML use case diagrams, activity diagrams describing the details of the use cases and a data model in the form of a simple class diagram.

The measurements were performed independently by two groups of measurers, each of which involved two measurement experts. The measurements were performed by different groups to better understand potential measurement variances caused by assumptions and interpretations of the different measurers. The results were verified according to the measurement rules of each method by the measurer who himself did not involved in that measurement process. In the group of measurers, one of them holds a PhD. degree in the related subjects; two are PhD. students and one is an MSc. student working on related subjects, in particular on software functional size measurement. All the measurers received training for at least one of the FSM methods and they all measured at least one project previously.

The measurement results are given in Table 2 and Table 3. Since ISO view takes the adjustment of the functional size via quality and technical requirements outside the scope of the FSM [13], we do not take into account the adjustment phase of the IFPUG FPA for the purpose of this case study.

It took 105 person-hours of effort to measure the functional size of KAMA implementing COSMIC FSM. The measurement with IFPUG FPA took 102 personhours. Although the functional size for each of the method differs significantly, the effort values utilized for the measurement were quite similar. 
Table 2. Case Project - IFPUG FPA Size Measurement Details

\begin{tabular}{ccccccc}
\hline $\begin{array}{c}\text { No. of } \\
\text { Elementary Processes }\end{array}$ & $\begin{array}{c}\text { No. of } \\
\text { ILFs }\end{array}$ & $\begin{array}{c}\text { No. of } \\
\text { EIFs }\end{array}$ & $\begin{array}{c}\text { No. of } \\
\text { EIs }\end{array}$ & $\begin{array}{c}\text { No. of } \\
\text { EOs }\end{array}$ & $\begin{array}{c}\text { No. of } \\
\text { EQs }\end{array}$ & $\begin{array}{c}\text { Functional Size } \\
\text { (IFPUG FP) } \\
\text { (Unadjusted) }\end{array}$ \\
\hline 45 & 11 & 0 & 26 & 1 & 18 & 306 \\
\hline
\end{tabular}

Table 3. Case Project - COSMIC FSM Size Measurement Details

\begin{tabular}{cccccc}
\hline $\begin{array}{c}\text { No. of } \\
\text { Functional Processes }\end{array}$ & $\begin{array}{c}\text { No. of } \\
\text { Entries }\end{array}$ & $\begin{array}{c}\text { No. of } \\
\text { Exits }\end{array}$ & $\begin{array}{c}\text { No. of } \\
\text { Reads }\end{array}$ & $\begin{array}{c}\text { No. of } \\
\text { Writes }\end{array}$ & $\begin{array}{c}\text { Functional Size } \\
\text { (COSMIC FP) }\end{array}$ \\
\hline 55 & 61 & 154 & 314 & 160 & 697 \\
\hline
\end{tabular}

\section{Findings and Conclusions}

Although the rules for identifying the BFCs and BFC types differ for each method, using similar concepts and comparable attributes, decomposition of the functional user requirements into 'transactions' is expected to yield the same set of transactions. While this is not explicitly asserted by any of the FSM methods, it is one of the underlying assumptions of research related with the conversion of the sizes or unification of these methods [8], [4], [10], [25]. When the same groups of measurers involve in the measurement process, they usually identify similar or identical transactions for measurements performed by different FSM methods [10], [11].

However, in this case study, the differences in the interpretation of the rules by different measurers cause significant differences in the measurement results. During the case study conduct, the measurers faced some difficulties in identifying the entities and transactions. This was mainly due to the structure of the data to be maintained by the application and the way the FSM methods handles entity generalization.

IFPUG Implementation Results. Although IFPUG FPA measurement process does not give any precedence rule for identifying the data and transactional functions, in our case study we started with the data functions. Because, we utilized ILFs to better identify the transactional functions and valuing their complexity. The complexity of a transactional function is dependent on the number of ILFs/EIFs maintained during the transactions as well as the total number of input and output DETs.

IFPUG FPA takes the complete inheritance hierarchy as a single logical file, with a RET for each subclass [12]. For example, the complete inheritance hierarchy of 'model elements' is considered as one ILF with a number of RETs for each special type (Fig. 2). Thus, with respect to the counting rules, the functional complexity of the model element ILF is high and so the contribution on the total functional size is 15 IFPUG function points (FP). The affect of number of RETs on functional size were limited in the sense that, with 10 RETs for each of the special model element type having attributes of their own, the contribution of the ILF is increased from 7 to 15 function points (complexity level from low to high). 


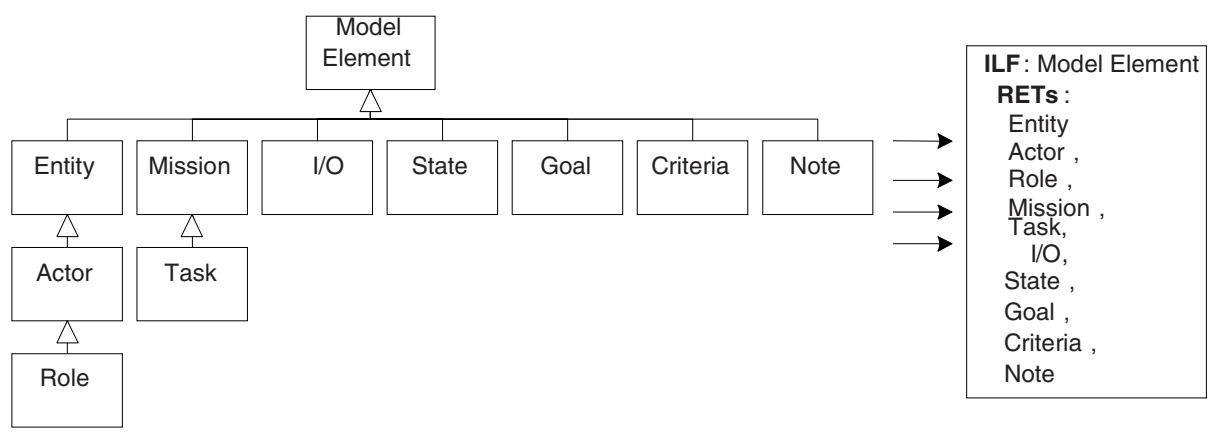

Fig. 2. A mapping from entities to an ILF in IFPUG FPA

Identifying the data functions (ILF \& EIF) was useful in determining the transactional functions, because the primary intend of the transactional functions (elementary processes in IFPUG FPA) is to maintain one or more logical files (create, update, read, delete, etc.). Besides, the functional complexity of a transactional function depends on the number of logical files referenced and the total number of input and output DET to and from the transaction.

Unifying all special entities in the inheritance hierarchy into an ILF also combined many of the transactions performed on each of the special entity. For example, a transaction of creating an 'actor' model element was combined with creating a 'state' model element, even though system may need to behave in a different way for each of them. It can be argued that those two entities are separate in the user domain and whether the application handles both entities in the same way or not can be a design choice rather than a decision to be given in the requirements phase.

The difference for those two cases can be significant for applications similar to KAMA, where entity abstractions (aggregation, generalization, etc) are applied extensively. For example, for the elementary process of creating a model element, the size is 6 FP (complexity level being high). On the other hand, having separate element creation process for each special type would result significantly larger values in total. For 10 specific types, the result would be $60 \mathrm{FP}$ (each having $6 \mathrm{FP}$ with functional complexity level high). Applying the same principle for other generalized entities (relationship and diagram types) and related elementary processes (update, deletion, read, etc.), the difference would be more substantial.

Based on these assumptions, where we consider each special type as a separate ILF, we re-measured the size and the resulting value turned out to be $1789 \mathrm{FP}$, as opposed to $306 \mathrm{FP}$ in the first measurement performed in the case study. The number of elementary processes increased from 45 to 260 and the number of ILFs increased from 11 to $41.485 \%$ difference in the functional size is significant.

Another notable difficulty about IFPUG FPA is related to the counting rules for transactional functions. One of the rules to be applied in order for an elementary process to be counted as a unique occurrence of an elementary process (external input-EI, external output-EO or external inquiry-EQ) is the following [12]:

"The set of data elements identified is different from the sets identified for other external inputs/outputs/inquiries for the application." 
In the context of entity generalization/specialization, this can be interpreted in a way which is different than the practices applied in the counting manual and other guiding sources [9]. For example, with respect to the practices applied regarding the rules in the counting manual, creating an 'actor' and 'state' model elements is considered as a unique external input maintaining the 'model element' ILF. However, with respect to the rule given above, we can argue that, if the 'actor' and 'state' model elements have different attributes other than the ones they have in common, creating each of them can be considered as different elementary processes. Because, creating an 'actor' model element will maintain a different set of DETs than creating the 'state' model element. This interpretation yet again may result considerable differences in the result. In order to observe the affect of such an interpretation on our case project, we recalculated the functional size. The resulting size value was $512 \mathrm{FP}$, which is $67 \%$ more than the original 306 FP value. The number of ILFs remained the same but the number of elementary processes increased from 45 to 82 . Hence, different interpretations and assumptions regarding the counting rules and the structure of the data leaded to differences in functional size, which was significant for our case.

COSMIC Implementation Results. For the COSMIC FSM measurement case, the measurement group had some difficulties particularly in identifying the functional processes and measuring their functional size. One of the main reasons for that was the lack of clear assistance in the measurement manual [28] for distinguishing processes that maintain a set of 'objects of interests' that can be abstracted to a general entity. The group's tendency was to treat all special entities as separate object of interests, and consider each transaction performed on them as separate functional processes. For example, two functional processes; creating a 'mission space' diagram and creating 'entity ontology' diagram are considered as separate since they maintain two different object of interests and they are triggered by different triggering events perceived by the functional user (triggering event 1 - the user wants to create an 'entity ontology' diagram; triggering event 2 - the user wants to create 'mission space' diagram). Accordingly, we obtained totally 270 functional processes. However, the processing logic of the functional processes which maintain sub-entities is the same. Therefore, we considered those as the same and measured only one. Based on this assumption, we obtained 55 functional processes in total, which was only $20 \%$ of the value obtained in first measurement.

Another difficulty was the measurement of each functional process which maintains sub-entities. The measurement group needed to refer to COSMIC FSM guideline for sizing business applications software [27]. The COSMIC FSM measurement manual [28] recommends the reader to refer to this guideline for the details on determining object of interests and separate data groups. To better handle generalization, the guideline introduces a new term; 'sub-type object of interest'.

According to the guideline, sub-types are the specialized entities (classes) that are in the lowest level in the inheritance hierarchy. The general principle is that where there is a need to distinguish more than one sub-type in the same functional process, each sub-type is treated as a separate object of interest. Hence, according to the rules in the guideline, instead of having separate functional processes for each special 
entity, their contribution on the functional size was taken into account by including additional data movements for each of the special entity (sub-type object of interest) in the functional processes. However, if the functional process did not need to distinguish special entities, only the general entity is referred. For example, creating a model element is a functional process that requires distinguishing each type of model element. For 10 special entities, there were 10 Entry and 10 Write data movements in the functional process.

COSMIC FSM guideline for sizing business applications software defines specific rules to handle entity generalizations in measuring the functional size of a functional process and provides examples demonstrating how generalizations can be reflected to the measurement practice. We still faced difficulties in identifying the functional processes maintaining sub-entities. Identifying functional processes are derived by the set of triggering events sensed by each of the functional user. In our case study, although their processing logics are similar, we arrived at separate functional processes each maintaining different sub-entities. Therefore, it is necessary to extend COSMIC FSM measurement manual with specific rules in order to clarify the procedure to be followed when identifying and combining similar functional processes which maintain sub-entities that are generalized. In addition, it is still arguable whether generalization or specialization practices can be performed in the user domain or they belong to the solution domain and are design issues.

In our study, we observed that, with different interpretations and assumptions, significantly different set of base functional components (BFCs) for the same software can be identified and this can occur not only among different FSM methods but also for the same method. We observed that there is an improvement opportunity for both methods regarding the rules to better accommodate entity generalizations, since current rules are subject to ambiguity and interpretation.

\section{References}

1. Abrahao, S., Poels, P., Pastor, O.: A Functional Size Measurement Method for ObjectOriented Conceptual Schemas: Design and Evaluation Issues. Software \& System Modeling 5(1), 48-71 (2006)

2. Albrecht, A.J.: Measuring Application Development Productivity. In: Proc. of the IBM Applications Development Symposium, Monterey, California, pp. 83-92 (1979)

3. Caldiera, G., Antoniol, G., Fiutem, R., Lokan, C.: Definition and Experimental Evaluation of Function Points for Object Oriented Systems. In: Proceedings of the 5th International Symposium on Software Metrics, Bethesda (1998)

4. Cuadrado-Gallego, J.J., Rodriguez, D., Machado, F., Abran, A.: Convertibility Between IFPUG and COSMIC Functional Size Measurements. In: Münch, J., Abrahamsson, P. (eds.) PROFES 2007. LNCS, vol. 4589, pp. 273-283. Springer, Heidelberg (2007)

5. DeMarco, T.: Controlling Software Projects. Yourdon press, New York (1982)

6. Diab, H., Frappier, M., St. Denis, R.: Formalizing COSMIC-FFP using ROOM. In: ACS/IEEE Inter. Conf. on Computer Systems and Applications, pp. 312-318 (2001) 
7. Fetcke, T., Abran, A., Nguyen, T.H.: Mapping the OO-Jacobson Approach into Function Point Analysis. In: Proceedings of TOOL 1997, Santa Barbara, CA (1998)

8. Fetcke, T., Abran, A., Dumke, R.: A Generalized Representation for Selected Functional Size Measurement Methods. In: International Workshop on Software Measurement (2001)

9. Garmus, D., Herron, D.: Measuring the Software Process: A Practical Guide to Functional Requirements. Prentice Hall, New Jersey (1996)

10. Gencel, C., Demirors, O.: Conceptual Differences Among Functional Size Measurement Methods. In: Proc. of the First International Symposium on Empirical Software Engineering and Measurement - ESEM 2007, Madrid, Spain, pp. 305-313 (2007)

11. Gencel, C., Demirors, O.: Functional Size Measurement Revisited. ACM Transactions on Software Engineering and Methodology (to be published, 2008)

12. International Function Point Users Group (IFPUG), Function Point Counting Practices Manual, Release 4.2.1 (2005)

13. IEEE Std. 14143.1: Implementation Note for IEEE Adoption of ISO/IEC 14143-1:1998 Information Technology- Software Measurement- Functional Size Measurement -Part 1: Definition of Concepts (2000)

14. ISO/IEC 14143-1: Information Technology - Software Measurement - Functional Size Measurement - Part 1: Definition of Concepts (1998, revised in 2007)

15. ISO/IEC TR 14143-5: Information Technology - Software Measurement - Functional Size Measurement - Part 5: Determination of Functional Domains for Use with Functional Size Measurement (2004)

16. ISO/IEC IS 20968:2002: Software Engineering - MK II Function Point Analysis Counting Practices Manual (2002)

17. ISO/IEC IS 20926:2003: Software Engineering - IFPUG 4.1 Unadjusted Functional Size Measurement Method - Counting Practices Manual (2003)

18. ISO/IEC 19761:2003: Software Engineering - COSMIC-FFP: A Functional Size Measurement Method (2003)

19. ISO/IEC IS 24570:2005: Software Engineering - NESMA functional size measurement method Ver.2.1 - Definitions and counting guidelines for the application of FPA (2005)

20. Jenner, M.S.: COSMIC-FFP and UML: Estimation of the Size of a System Specified in UML - Problems of Granularity. In: Proc. the Fourth European Conference on Software Measurement and ICT Control, pp. 173-184 (2001)

21. Jones, T.C.: A Short History of Function Points and Feature Points. Software Productivity Research Inc., USA (1987)

22. NESMA, Definitions and Counting Guidelines for the Application of Function Point Analysis, Version 2.0 (1997)

23. OMG, Unified Modeling Language: Superstructure, Ver.2.0, Formal/05-07-04, Object Management Group (2005)

24. Rains, E.: Function points in an Ada object-oriented design? OOPS Messenger 2(4), 23 25 (1991)

25. Symons, C.: Software Sizing and Estimating: MkII Function Point Analysis. John Wiley, Chichester (1993)

26. Symons, C.: Come Back Function Point Analysis (Modernized) - All is Forgiven!). In: Proc. of the 4th European Conf. on Software Measurement and ICT Control (FESMADASMA 2001), Germany, pp. 413-426 (2001)

27. The Common Software Measurement International Consortium (COSMIC): Guideline for Sizing Business Applications Software Using COSMIC-FFP, Version 1.0 (2005)

28. The Common Software Measurement International Consortium (COSMIC): COSMIC Method, Version 3.0, Measurement Manual (2007) 
29. The United Kingdom Software Metrics Association: MkII Function Point Analysis Counting Practices Manual, V.1.3.1 (1998)

30. Whitmire, S.A.: 3D Function Points: Scientific and Real-time Extensions to Function Points. In: Proceedings of the 1992 Pacific Northwest Software Quality Conference (1992)

31. Whitmire, S.A.: Applying function points to object-oriented software models. In: Keyes, J. (ed.) Software Engineering Productivity Handbook, pp. 229-244. McGraw-Hill, New York (1992) 